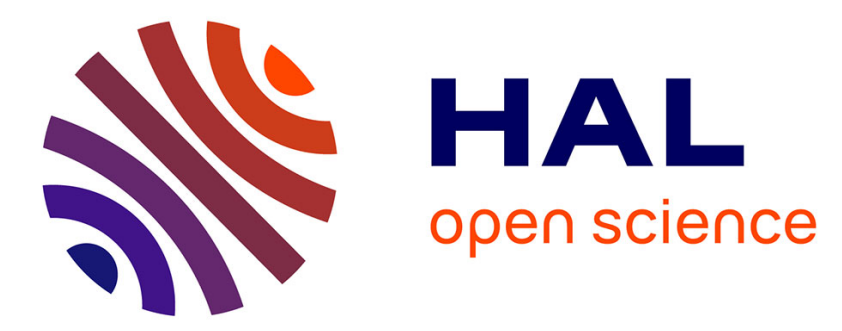

\title{
Spectroscopic study of the incommensurate phase of ThBr4 via the optical and magnetooptical properties of $\mathrm{U} 4+$
}

B. Briat, P. Delamoye, J.C. Rivoal, S. Hubert, P. Evesque

\section{- To cite this version:}

B. Briat, P. Delamoye, J.C. Rivoal, S. Hubert, P. Evesque. Spectroscopic study of the incommensurate phase of ThBr4 via the optical and magnetooptical properties of U4+. Journal de Physique, 1985, 46 (8), pp.1375-1386. 10.1051/jphys:019850046080137500 . jpa-00210081

\section{HAL Id: jpa-00210081 https://hal.science/jpa-00210081}

Submitted on 1 Jan 1985

HAL is a multi-disciplinary open access archive for the deposit and dissemination of scientific research documents, whether they are published or not. The documents may come from teaching and research institutions in France or abroad, or from public or private research centers.
L'archive ouverte pluridisciplinaire HAL, est destinée au dépôt et à la diffusion de documents scientifiques de niveau recherche, publiés ou non, émanant des établissements d'enseignement et de recherche français ou étrangers, des laboratoires publics ou privés. 
Classification

Physics Abstracts

$78.20 \mathrm{~L}-64.70 \mathrm{~K}-78.50-78.40$

\title{
Spectroscopic study of the incommensurate phase of $\mathbf{T h B r}_{4}$ via the optical and magnetooptical properties of $\mathbf{U}^{4+}$
}

\author{
B. Briat $\left({ }^{*}\right)$, P. Delamoye $\left({ }^{+}\right)$, J. C. Rivoal $\left({ }^{*}\right)$, S. Hubert $\left({ }^{+}\right)$and P. Evesque $(*)$ \\ (*) Laboratoire d'Optique Physique, ESPCI, 10, rue Vauquelin, 75231 Paris Cedex 05, France \\ $\left(^{+}\right)$Laboratoire de Radiochimie, Institut de Physique Nucléaire, Université de Paris-Sud, B.P. 1, 91406 Orsay, \\ France
}

(Reçu le 19 décembre 1984, accepté le 22 mars 1985)

\begin{abstract}
Résumé. - $\mathrm{U}^{4+}$ est utilisé pour tester la phase incommensurable de $\mathrm{ThBr}_{4}$ grâce à des mesures d'absorption et de dichroïsme circulaire magnétique. En dessous de $T_{\mathrm{c}}=95 \mathrm{~K}$, la symétrie au site de l'ion sonde est $\mathrm{D}_{2 d}$ ou $\mathrm{D}_{2}$; elle peut être simulée en faisant varier un angle de phase $\varphi$ entre $0\left(D_{2 d}\right)$ et $\pm \pi / 2\left(D_{2}\right)$. Le DCM présente sur l'absorption le grand avantage de montrer une singularité pour les centres $D_{2 d}$. Nous présentons un modèle permettant de reproduire assez fidèlement nos courbes d'absorption, d'effet Zeeman et de DCM. Nous aboutissons à deux conclusions importantes : (i) la phase est probablement vraiment incommensurable dans la mesure où nous établissons à 80 une limite inférieure du nombre de sites différents; (ii) pour un cristal de concentration $2 \times 10^{-4}$, nous établissons l'absence d'accrochage de l'onde incommensurable sur les impuretés. Par ailleurs, le DCM démontre sans ambiguité que l'état fondamental de $\mathrm{U}^{4+}$ dans $\mathrm{ThBr}_{4}$ est $\Gamma_{4}$ en symétrie $\mathrm{D}_{2 d}$. Nous déterminons enfin le facteur de Landé de cinq niveaux excités, ces mesures étant en bon accord avec les résultats de calculs récents.
\end{abstract}

\begin{abstract}
U}^{4+}$ is used to probe the incommensurate structure of $\mathrm{ThBr}_{4}$ via absorption and magnetic circular dichroism (MCD) measurements. Below $T_{c}=95 \mathrm{~K}$, the crystal field experienced by $\mathrm{U}^{4+}$ ions is either of $\mathrm{D}_{2 d}$ or $\mathrm{D}_{2}$ symmetry. This can be simulated by varying a phase angle $\varphi$ form $0\left(D_{2 d}\right)$ to $+\pi / 2\left(D_{2}\right)$. One very neat advantage of MCD over absorption is that the $\mathrm{D}_{2 d}$ centres show up very clearly in the former due to the occurrence of a derivative-like singularity. We present a model to simulate absorption, Zeeman and MCD profiles. It accounts satisfactorily for most of our data and we arrive at the following main conclusions : (i) it is probable that the phase is truly incommensurate since the lower limit of the number of sites is found to be 80 ; (ii) for a low concentration of $\mathrm{U}^{4+}\left(2 \times 10^{-4}\right)$, we found no evidence of a pinning of the incommensurate modulation by the impurities. MCD provides also a definite proof that the ground state of $\mathrm{U}^{4+}$ in $\mathrm{ThBr}_{4}$ is $\Gamma_{4}$ with $\mathrm{D}_{2 d}$ symmetry. Finally, we determine the Landé factors of five excited states, our findings being in fair agreement with the results of recent crystal field calculations.
\end{abstract}

\section{Introduction.}

Seven years ago [1], $\mathrm{ThBr}_{4}$ was only considered as an additional suitable host for spectroscopic studies on tetravalent actinide ions. At room temperature, the crystal is tetragonal with space group $\mathrm{D}_{4 \mathrm{~h}}^{19}$. As for $\mathrm{Zr}^{4+}$ in zircon $[2,3]$, each $\mathrm{Th}^{4+}$ ion is located at a site of $\mathrm{D}_{2 d}$ symmetry. Due to the lack of an inversion centre, absorption spectra of doped crystals were expected to show parity allowed electric dipole origins, the location of which was to be compared with the results of crystal-field calculations. The situation is substantially different since the discovery [4] that $\mathrm{ThBr}_{4}$ is a prototype incommensurate (INC) material below $T_{\mathrm{c}}=95 \mathrm{~K}$ and is probably so all the way down to zero Kelvin. One aim of present studies is probably to probe the INC structure by, e.g., spectroscopic means or else to examine the effect of impurities on this structure. Earlier low-temperature absorption and emission spectra [1] of $\mathrm{ThBr}_{4} / \mathrm{U}^{4+}$ have been reexamined and a model has been presented to explain the unusual line-shapes encountered [5]. Basically, in the INC phase there is a transverse sinusoidal displacement of the bromide ions perpendicular to the $c$-axis of the crystal and the crystal field probed by $\mathrm{U}^{4+}$ is either $\mathrm{D}_{2 d}$ or $\mathrm{D}_{2}$. This model has recently been checked further against site-selective excitation measurements [6] and theoretical calculations [7]. All experimental data available support a continuous distribution of crystal-field strengths at the $\mathrm{U}^{4+}$ sites. 
The present study has been undertaken with several aims in mind :

(i) to provide an experimental and unambiguous proof of the long postulated singlet character of the ground state of $\mathrm{U}^{4+}$ in $\mathrm{ThBr}_{4}$. For that purpose, polarized absorption studies (axial, $\pi$ and $\sigma$ ) have been complemented by magnetic circular dichroism (MCD) measurements. It is well established [8] that MCD can easily distinguish between a diamagnetic and a paramagnetic species. Moreover, the sign of the observed features can be correlated with the nature of the ground and excited states;

(ii) to derive (via $\mathrm{MCD}$ ) the spectroscopic splitting factors of the $\mathrm{U}^{4+}$ excited states for a severe test of the correctness of computed wave functions ;

(iii) to develop more fully and check further the model outlined previously, by predicting the profile of MCD spectra and comparing with experimental findings.

In the next section, $\S 2$, we present our model for the simulation of absorption and MCD profiles. In $\S 3$ we describe our experimental conditions and the more important results. These data are finally discussed in $\S 4$.

\section{Theoretical model.}

\subsection{ABSORPTION AND MCD.}

2.1.1 $\mathrm{D}_{2 d}$ symmetry. - We first ignore the existence of an INC phase and establish selection rules for electric dipole transitions in the case of an even electron configuration $\left(\mathrm{f}^{2}\right)$. Those for a $\mathrm{D}_{2 d}$ symmetry [9] are given in table Ia where $\sigma$ and $\pi$ refer to the electric vector of the light beam being perpendicular and parallel to the tetragonal $c(z)$ axis respectively. The axial $(\alpha)$ and $\sigma$ spectra are expected to be similar. In the presence of a magnetic field $B$ along $z$, the symmetry is lowered to $\mathrm{S}_{4}$. Then $\Gamma_{1}, \Gamma_{2} \rightarrow \Gamma_{1}^{\prime} ; \Gamma_{3}, \Gamma_{4} \rightarrow \Gamma_{2}^{\prime}$ and $\Gamma_{5} \rightarrow \Gamma_{3}^{\prime}+\Gamma_{4}^{\prime}$ where primes are used to distinguish $\mathrm{S}_{4}$ from $\mathrm{D}_{2 d}$ notations. The new selection rules are also shown in table $\mathrm{Ib}$, where $\sigma_{+}$and $\sigma_{-}$stand for circularly polarized light travelling along $z(\alpha$ spectrum) and are associated with the operators $m_{ \pm}=\mp$ $(i / \sqrt{2})\left(m_{x} \pm m_{y}\right)$ which transform as $\Gamma_{4}^{\prime}\left(m_{+}\right)$and $\Gamma_{3}^{\prime}\left(m_{-}\right)$.

MCD is defined as the difference in absorbance $\Delta A=A_{+}-A_{-}$for $\sigma_{+}$and $\sigma_{-}$light. In $\mathrm{D}_{2 d}$ (Table Ia), this can only occur for a singlet $\rightarrow$ doublet or a doublet $\rightarrow$ singlet transition between the ground state $\left|g_{0}\right\rangle$ and the excited state $\left|e_{0}\right\rangle$. In the limit where the Zeeman splitting is small compared to both the thermal energy $k T$ and half the line-width, the rigid shift approximation leads to [8] :

$$
\Delta A / E=K \mu_{\mathrm{B}} B\left\{\mathcal{A}_{1}(-\partial f / \partial E)+\left(\mathfrak{B}_{0}+\mathcal{C}_{0} / k T\right) f\right\}
$$

while $A / E=K f \mathfrak{D}_{0}$ for a zero magnetic field.
Table I. - Electric dipole transitions for even electron configurations. $\mathrm{g}_{0}$ and $\mathrm{e}_{0}\left(\mathrm{~g}_{0}^{\prime}\right.$ and $\left.\mathrm{e}_{0}^{\prime}\right)$ stand for the ground and excited states respectively.

\begin{tabular}{|c|ccccc|}
\hline \multicolumn{1}{c|}{$\mathrm{D}_{2 d}$} \\
\hline $\mathrm{g}_{0}$ & $\Gamma_{1}$ & $\Gamma_{2}$ & $\Gamma_{3}$ & $\Gamma_{4}$ & $\Gamma_{5}$ \\
\hline$\Gamma_{1}$ & & & & $\pi$ & $\sigma$ \\
\hline$\Gamma_{2}$ & & & $\pi$ & & $\sigma$ \\
\hline$\Gamma_{3}$ & & $\pi$ & & & $\sigma$ \\
\hline$\Gamma_{4}$ & $\pi$ & & & $\sigma$ \\
\hline$\Gamma_{5}$ & $\sigma$ & $\sigma$ & $\sigma$ & $\sigma$ & $\pi$ \\
\hline
\end{tabular}

$\underline{b-S_{4}}$

\begin{tabular}{|c|c|c|c|c|}
\hline $\mathrm{e}_{0}^{\prime}$ & $\Gamma_{1}^{\prime}$ & $\Gamma_{2}^{\prime}$ & $\Gamma_{3}^{\prime}$ & $\Gamma_{4}^{\prime}$ \\
\hline$\Gamma_{1}^{\prime}$ & & $\pi$ & $\sigma_{+}$ & $\sigma_{-}$ \\
\hline$\Gamma_{2}^{\prime}$ & $\pi$ & & $\sigma_{-}$ & $\sigma_{+}$ \\
\hline$\Gamma_{\mathbf{3}}^{\prime}$ & $\sigma_{-}$ & $\sigma_{+}$ & & $\pi$ \\
\hline$\Gamma_{4}^{\prime}$ & $\sigma_{+}$ & $\sigma_{-}$ & $\pi$ & \\
\hline
\end{tabular}

$f$ is a normalized shape function $\left(\int f \mathrm{~d} E=1\right), E$ is the energy (in wave numbers hereafter); $D_{0}$ is the dipole strength of the transition; $K$ is a numerical factor and $\mu_{\mathrm{B}}$ is the Bohr-magneton. The $\mathcal{C}_{0}$ term arises from the difference in population among the possible substates of $\left|g_{0}\right\rangle$ in $S_{4}$, i.e., it can be nonzero only when $\left|g_{0}\right\rangle=\Gamma_{5}$. The origin of the $\mathcal{A}_{1}$ term lies in the energy differences between the two Zeeman components. This term should therefore be observable in principle for all $\sigma$ transitions of $\mathrm{U}^{4+}$. $\mathfrak{B}_{0}$ arises from the possible mixing of $\left|g_{0}\right\rangle$ or/and $\left|\mathrm{e}_{0}\right\rangle$ with a state $\left|\mathrm{k}_{0}\right\rangle$ located at an energy $\Delta W$ above it. Since $B$ transforms as $\Gamma_{1}^{\prime}$ in $S_{4}, \mathfrak{B}_{0}$ terms are expected only for those representations of $D_{2 d}$ which reduce to the same representation in $\mathrm{S}_{4}$, namely $\Gamma_{1}-\Gamma_{2}, \Gamma_{3}-\Gamma_{4}$ and $\Gamma_{5}-\Gamma_{5}$. Note that if the coupling arises between $\left|\mathrm{e}_{0}\right\rangle$ and $\left|\mathrm{k}_{0}\right\rangle$, the transitions $\left|g_{0}\right\rangle \rightarrow\left|e_{0}\right\rangle$ and $\left.\left|g_{0}\right\rangle \rightarrow k_{0}\right\rangle$ show $\mathfrak{B}_{0}$ terms of the same absolute magnitude but of opposite sign. Finally, the peak to peak magnitude of the derivativelike term in equation (1) varies approximately as $1 / \gamma$ while the two absorption-like contributions are proportional to $1 / \Delta W$ and $1 / k T$ respectively. 
MCD parameters are usually extracted via a moment analysis of the data. For further use, we define :

$$
\begin{aligned}
\langle A\rangle_{0} & =\int(A / E) \mathrm{d} E=K \mathscr{D}_{0}, \\
\bar{E} & =\left(1 /\langle A\rangle_{0}\right) \int A \mathrm{~d} E \\
\langle\Delta A\rangle_{0} & =\left(1 /\langle A\rangle_{0}\right) \int(\Delta A / E) \mathrm{d} E \\
\langle\Delta A\rangle_{1} & =\left(1 /\langle A\rangle_{0}\right) \int(\Delta A / E)(E-\bar{E}) \mathrm{d} E
\end{aligned}
$$

where $\bar{E}$ is the barycentre of the line.

Assuming first a non-degenerate ground state and an isolated $\mathrm{s} \rightarrow \mathrm{d}$ transition, we easily find $\langle\Delta A\rangle_{0}=0$ and $\langle\Delta A\rangle_{1}= \pm 2 g \mu_{\mathrm{B}} B$. Here we adopt the convention $\left(^{1}\right)\left\langle\Gamma_{3}^{\prime}\left|L_{z}+2 S_{z}\right| \Gamma_{3}^{\prime}\right\rangle=g$, the plus or minus sign depending on whether the ground state is $\Gamma_{2}^{\prime}$ or $\Gamma_{1}^{\prime}$ in $\mathrm{S}_{4}$ symmetry. Knowledge of the nature of the ground state and the sign of the associated $\langle\Delta A\rangle_{1}$ would lead to the sign of the $g$ factors for the excited doublets $\left(\mathrm{D}_{2 d}\right)$. We note that the relationship between $\langle\Delta A\rangle_{1}$ and $g$ does not require the rigid shift approximation and is independent of electron-phonon coupling. If the ground state were a doublet, then $\langle\Delta A\rangle_{0}$ would carry the information regarding the sign of its $g$ factor and the symmetry of the excited state.

2.1.2 $\mathrm{D}_{2}$ symmetry. - Anticipating upon our discussion, we treat the MCD problem for the case of a $\Gamma_{4} \rightarrow \Gamma_{5}$ transition in $\mathrm{D}_{2 d}$. This has been done previously in the case of $\mathrm{Pr}^{3+}$ in $\mathrm{AlLaO}_{3}$ [10]. Let $\mid \mathrm{s}>$ be a $\Gamma_{2}^{\prime}$ ground state in the presence of $B,|\mathrm{p}\rangle$ and $|\mathrm{q}\rangle$ the excited states associated with $\Gamma_{3}^{\prime}$ and $\Gamma_{4}^{\prime}$ respectively. Then $\left|\left\langle\mathrm{p}\left|m_{+}\right| \mathrm{s}\right\rangle\right|=\left|\left\langle\mathrm{q}\left|m_{-}\right| \mathrm{s}\right\rangle\right|$ (Table Ib). For convenience, we take 1 as their common value. In the absence of $B$, for $\mathrm{D}_{2}$ symmetry, the $\Gamma_{5}$ doublet splits into two singlets separated by an energy $2 \Delta$. Appropriate wavefunctions are now of the form :

$$
\begin{aligned}
& |\mathrm{P}\rangle=\alpha|\mathrm{p}\rangle+\beta|\mathrm{q}\rangle \\
& |\mathrm{Q}\rangle=\beta^{*}|\mathrm{p}\rangle-\alpha^{*}|\mathrm{q}\rangle
\end{aligned}
$$

where one can take $\alpha=\beta=1 / \sqrt{2}$ without loss of generality. Then, $|\mathrm{P}\rangle$ and $|\mathrm{Q}\rangle$ transform as $y$ and $-i x$ respectively in $D_{2}$ symmetry. When $B$ is applied, the Hamiltonian is :

$$
\begin{aligned}
& |\mathrm{P}\rangle \quad|\mathrm{Q}\rangle \\
& \begin{array}{l}
|\mathrm{P}\rangle \\
|\mathrm{Q}\rangle
\end{array}\left(\begin{array}{cc}
\Delta & g \mu_{\mathrm{B}} \mathrm{B} \\
g \mu_{\mathrm{B}} B & -\Delta
\end{array}\right)
\end{aligned}
$$

with eigenvalues $\pm \lambda$ where $\lambda=\left[\Delta^{2}+\left(g \mu_{\mathrm{B}} B\right)^{2}\right]^{1 / 2}$.

(1) $\Gamma_{3}^{\prime}$ states are associated with wavefunctions of the general form $\Sigma a_{i} \mid L S J M ; M=5,1$ or -3$\rangle$.

JOURNAL DE PHYSIQUE. - T. 46, № 8, AOOT 1985
The associated eigenfunctions are :

$$
\begin{aligned}
& |+\rangle=a^{-1 / 2}[|\mathrm{P}\rangle+b|\mathrm{Q}\rangle] \\
& |-\rangle=-a^{-1 / 2}[-b|\mathrm{P}\rangle+|\mathrm{Q}\rangle]
\end{aligned}
$$

where $a=2 \lambda /(\lambda+\Delta)$ and $b=g \mu_{\mathrm{B}} B /(\lambda+\Delta)$.

So, for example, $\left|\left\langle+\left|m_{ \pm}\right| \mathrm{s}\right\rangle\right|^{2}=(1 / 2 a)(1 \pm b)^{2}$ and $\langle\Delta A\rangle_{0}$ for the $|\mathrm{s}\rangle \rightarrow| \pm\rangle$ transitions is $\pm g \mu_{\mathrm{B}} B / \lambda$. One has the situation of two states mixed via the magnetic field and giving rise to opposite $\Re_{0}$ terms with an absorption like shape.

2.2 InCOMmensurate PHASE. - Below $T_{\mathrm{c}}=95 \mathrm{~K}$, neutron diffraction experiments [4] indicate a modulated structure for $\mathrm{ThBr}_{4}$, with the modulation wavevector $q_{\mathrm{s}}$ being directed along the tetragonal axis :

$$
q_{\mathrm{s}}=(2 \pi / c) \zeta_{\mathrm{s}} \text { with } \zeta_{\mathrm{s}}=0.310 \pm 0.005
$$

The consequences of this structural change upon absorption line-shapes have already been outlined by Delamoye [5]. We provide here a more general and quantitative treatment with the added consideration of the action of a magnetic field.

For the dilution levels used in our experiments, the $\mathrm{U}^{4+}$ ions may be treated as isolated impurities, randomly distributed throughout the host lattice. Each of them is surrounded by two distorted tetrahedra of $\mathrm{Br}^{-}$ions. Below $T_{\mathrm{c}}$, each of $8 \mathrm{Br}^{-}$ions (index $i$ ) suffers a displacement $u_{i}$ along one or the other of two orthogonal directions perpendicular to the tetragonal axis. $u_{i}$ can be written as :

$$
u_{i}=\eta \cos \left(\dot{\varphi}+\varphi_{i}\right)
$$

where $\eta$ is a temperature dependent amplitude factor which may be taken as the order parameter; $(-\pi / 2 \leqslant \varphi \leqslant \pi / 2)$ is a linear function of the $z$ coordinate and characterizes a given $\mathrm{U}^{4+}$ ion. The various $\varphi_{i}$ are known from crystallographic data. It can be shown [5] that $\mathrm{U}^{4+}$ ions lie at a site of symmetry $\mathrm{D}_{2 d}(\varphi=0)$ or $\mathrm{D}_{2}(\varphi \neq 0)$.

We have derived formally the explicit dependence of the crystal field parameters upon the displacements of the $\mathrm{Br}^{-}$ions. After tedious calculations, one obtains the energies $\Delta(\varphi)$ of the various levels under the general form :

$$
\Delta(\varphi)=\Delta_{0}+\eta^{2}\left(\alpha+\beta \sin ^{2} \varphi\right) \pm \gamma \eta \sin \varphi
$$

where the coefficients $\alpha, \beta$ and $\gamma$ are all (in principle) non-zero in the case of a " high temperature " doublet while $\gamma=0$ for a $\mathbf{D}_{2 d}$ singlet. Equation (2) can also be found by using solely symmetry arguments. A comparable approach was used previously by Blinc et al. [11] to discuss their NMR data. Since optical spectroscopy concerns differences in energy $E(\varphi)$ between the ground state and the various excited states, we adopt here the following notation :

$$
E(\varphi)=E_{0} \pm v_{1} \sin \varphi+\left(v_{2} / 2\right) \sin ^{2} \varphi .
$$


We can now express the inhomogeneous line-shape of the composite absorption spectrum, or the absorbance at energy $E$ as :

$$
A / E \sim \int L[E, E(\varphi)] n \mathrm{~d} E
$$

where $n$ is the density of spectral lines at the frequencies $E(\varphi)$ and $L[E, E(\varphi)]$ is a normalized shape function corresponding to the absorption of $\mathrm{U}^{4+}$ in a given site.

If $\mathrm{d} N$ stands for the number of atoms absorbing at an energy lying in the range $\mathrm{d}[E(\varphi)]$ around $E(\varphi)$, then :

$$
n \mathrm{~d} E=\mathrm{d} N=(\mathrm{d} N / \mathrm{d} z)(\mathrm{d} z / \mathrm{d} \varphi) \mathrm{d} \varphi .
$$

Since the distribution is random $(\mathrm{d} N / \mathrm{d} z=$ constant $)$ and $\varphi$ is a linear function of $z$, one has $n \mathrm{~d} E=\mathrm{d} \varphi$ and :

$$
A / E \sim L[E, E(\varphi)] \mathrm{d} \varphi
$$

$A / E$ will present singularities whenever $n$ is maximum, i.e., when $\mathrm{d}[E(\varphi)] / \mathrm{d} \varphi=0$. For a singlet $\leftrightarrow$ singlet transition, two singularities with equal intensity are predicted for $|\varphi|=\pi / 2\left(E_{+}=E_{-}=E_{0}+v_{2} / 2\right)$ and $\varphi=0\left(E=E_{0}\right)$. In the case of a singlet doublet transition, the absorption profile also shows two singularities $(\varphi= \pm \pi / 2)$ at $E_{ \pm}=E_{0} \pm v_{1}+v_{2} / 2$ when $k=\left|v_{2} / v_{1}\right|<1$. A third one is predicted at $E_{\mathrm{c}}=E_{0}+\left(v_{1}\right)^{2} / 2 v_{2}$ for $\sin \varphi=\mp 1 / k$ when $|k|>1$. Note that, when present, the extra band at $E_{\mathrm{c}}$, always lies outside the interval between $E_{+}$and $E_{-}$. Furthermore, upon increasing the energy, the relative intensities of the three bands either increase or decrease continuously. An important result is that no singularity is expected for $\varphi=0$, i.e., for those centres at a site of $\mathrm{D}_{2 d}$ symmetry. The frequency separation between $E_{+}$and $E_{-}$is predicted to vary with temperature since :

$$
E_{+}-E_{-}=2 v_{1} \sim \eta \sim\left(T_{\mathrm{c}}-T\right)^{\beta}
$$

where $\beta$ is the critical exponent of the order parameter.

A program has been written to compute absorption, Zeeman effect and MCD profiles for a model including in $\mathrm{D}_{2 d}$ a singlet ground state and two excited doublets, one of which (or one of its $D_{2}$ branches) being thermally populated. The following notations were adopted :

Ground state

$$
E(\varphi)=0
$$

Low lying doublet

$$
E_{ \pm 1}=E_{0}^{1}+\left(v_{2}^{1} / 2\right) \sin ^{2} \varphi \pm \lambda_{1} .
$$

Higher doublet

$$
E_{ \pm 2}=E_{0}^{2}+\left(v_{2}^{2} / 2\right) \sin ^{2} \varphi \pm \lambda_{2}
$$

with

$$
\begin{array}{ll}
\lambda_{1}=\left[\left(v_{1}^{1} \sin \varphi\right)^{2}+\left(z_{1}\right)^{2}\right]^{1 / 2}, & z_{1}=g_{1} \mu_{\mathrm{B}} B \\
\lambda_{2}=\left[\left(v_{1}^{2} \sin \varphi\right)^{2}+\left(z_{2}\right)^{2}\right]^{1 / 2}, & z_{2}=g_{2} \mu_{\mathrm{B}} B .
\end{array}
$$

Putting $v_{1}^{2}=z_{2}=0$ corresponds to the problem of a singlet $\rightarrow$ singlet transition.

The first step is to compute for all $\varphi$ values $(-\pi / 2 \leqslant$ $\varphi \leqslant \pi / 2$ ) the respective populations $P_{i}$ (Boltzmann distribution) of the levels at $E_{i}(i=0,+1$ and -1$)$. Next, we assume normalized Lorentzian lines $L(E, E(\varphi))=(1 / \pi \gamma) /\left(1+X^{2}\right)$ with $X=\left(E-E_{i}\right) / \gamma$. For a singlet $\rightarrow$ doublet transition $\left(\mathrm{D}_{2 d}\right.$ notation), one finally obtains the Zeeman effect and MCD profiles from the following relationships :

$$
\begin{aligned}
A / E & \propto(1 / 2) \int\left[L\left(E, E_{+2}\right)+L\left(E, E_{-2}\right)\right] P_{0} \mathrm{~d} \varphi \\
\Delta A / E & \propto \int\left[L\left(E, E_{+2}\right)-L\left(E, E_{-2}\right)\right]\left(z_{2} / \lambda_{2}\right) P_{0} \mathrm{~d} \varphi
\end{aligned}
$$

Absorption profiles are found by simply putting $z_{1}=z_{2}=0$.

Figure 1 illustrates the results of such calculations $(\mathrm{d} \varphi=\pi / 100)$ in the case where the first doublet is ignored. One very neat qualitative advantage of $\mathrm{MCD}$ versus absorption is that the $\mathrm{D}_{2 d}$ centre $(\varphi=0)$ shows up very clearly in the former, due to the occurrence of

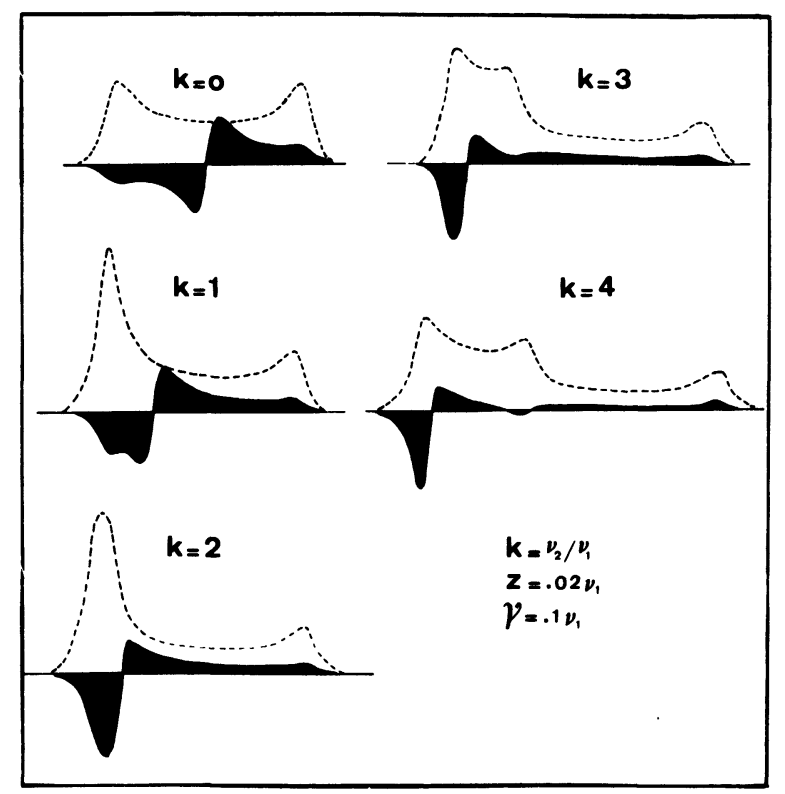

Fig. 1. - Illustration of the simulated absorption (doted line) and MCD (full line) profiles in the case of an isolated $\mathrm{s} \rightarrow \mathrm{d}$ transition, as a function of $k=v_{2} / v_{1}$. We chose $z_{2}=0.02 v_{1}, \gamma=0.1 v_{1}$ and $v_{1}>0$. For $k$ positive, the energy increases from left to right (right to left for $k<0$ ). 
the associated $\mathcal{A}_{1}$ term. As $|k|$ increases from 0 , this term moves from the centre of the spectrum towards one of the ends since $E_{\mathrm{c}}$ is hardly distinguishable from $E_{0}$ when $|k| \geqslant 3$. We note that, irrespective of the value and sign of $v_{1}^{2}$ and $v_{2}^{2},\langle\Delta A\rangle_{1}$ is always $2 z_{2}$ i.e., the value found in the case of one centre in $\mathrm{D}_{2 d}$ symmetry.

Without loss of generality, we can always state that $v_{1}>0$ and so only change the sign of $v_{2}$ to simulate all possible situations. Only slight modifications in equations (6), (7) are necessary in order to compute profiles for the hot bands associated with doublet $\rightarrow$ singlet transitions $\left(\mathrm{D}_{2 d}\right.$ nomenclature).

\section{Experimental.}

3. 1 EQUIPMENT. - Absorption and MCD data have been taken on five crystals separated from different lingots, of thickness $t$ ranging from 0.54 to $2.6 \mathrm{~mm}$ and concentration $c(\mathrm{U} / \mathrm{Th})$ of the order of $10^{-3}-10^{-4}$. All quantitative data will be given for a crystal of $t=0.54 \mathrm{~mm}$ and chemically determined $c=2 \times 10^{-4}$. A spectral band pass of $0.3 \AA$ was found to be appropriate to get all the details of the $\sigma, \pi$ and MCD spectra.

$\mathrm{ThBr}_{4} / \mathrm{U}^{4+}$ crystals prepared as described in [12] are sensitive to moisture and are highly birefringent. Special care was required for MCD measurements since a slight misorientation of the crystal, especially when combined with a defective surface leads to a significant depolarization of the light beam and thus to an important reduction of the MCD signal (or even artefacts). Data were taken on platelets cleaved perpendicularly to the tetragonal axis, checked under the polarizing microscope and freshly polished under a stream of dry hot air. We verified the absence of stray signal in zero field and corrected the data for the rate of depolarization as measured by placing an optically active sample (camphorquinone in dioxane) before and after the crystal investigated. MCD spectra were taken with standard equipment, i.e., a photoelastic modulator [13] and a phase sensitive detector. Measurements at $4.2 \mathrm{~K}$ and below (pumped helium) were made with a superconducting magnet $(2.5 \mathrm{~T}$ or $5 \mathrm{~T})$. All quantitative data are normalized to $1 \mathrm{~T}$. Less care was required to obtain reliable axial $\sigma$ and $\pi$ absorption spectra over a wide range of temperatures.

3.2 MAIN RESUlts. - Figure 2 shows a summary of our polarized absorption and MCD spectra in the $14200-22100 \mathrm{~cm}^{-1}$ spectral range. Quite noticeable is the fact that a number of sharp features occur on the top of a poorly defined broad band structure. This occurs especially around $17350 \mathrm{~cm}^{-1}$. Up to $2.5 \mathrm{~T}$, the height of MCD peaks was found to vary linearly with the applied magnetic field.

The $\sigma$ and axial spectra were found to be identical, thus demonstrating the electric dipole character of transitions. More detailed data for selected spectral

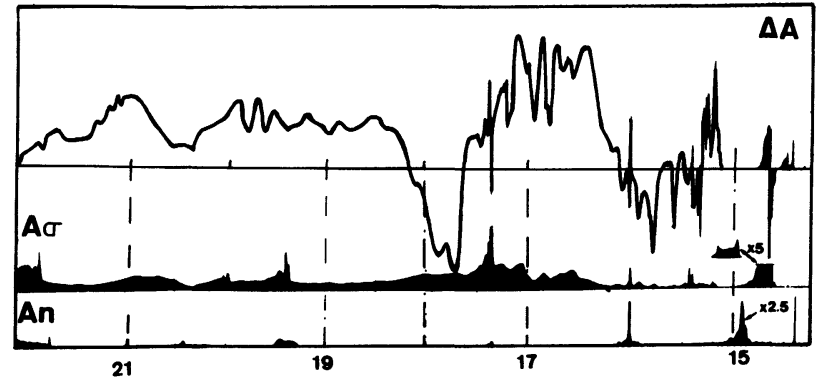

Fig. 2. - Schematic view of our MCD and polarized absorption spectra. All scales are arbitrary. $T=2.1 \mathrm{~K}$.

ranges are shown in subsequent figures and will now be briefly commented upon.

$$
\text { - } 14580-14730 \mathrm{~cm}^{-1} \text { (Fig. 3). }
$$

The strongest axial absorption is found in this region and extends over more than a hundred wavenumbers. The extreme bands $\alpha$ and $\beta$ are separated by $92 \mathrm{~cm}^{-1}$, with an unresolved shoulder on the blue side of $\alpha$. Additional features occur such as band $\delta$ and a quite intringuing dip $\gamma$.

The associated MCD was reproduced for three different samples where in one case the positive bump around band $\beta$ was replaced by a dip going to $\Delta A=0$. At both $4.2 \mathrm{~K}$ and $1.7 \mathrm{~K},\langle\Delta A\rangle_{0}$ was found to be strictly zero, a result inconsistent with a paramagnetic ground state in $\mathrm{D}_{2 d}$.

$$
\text { - } 15390-15450 \mathrm{~cm}^{-1} \text { (Fig. 4). }
$$

We always observed two sharp peaks $\alpha$ and $\beta$ $\left(E_{\alpha}-E_{\beta}=30 \mathrm{~cm}^{-1}\right)$ in the axial spectrum while the additional features marked by arrows were found to depend upon the sample, and are clearly seen in the MCD spectrum.

$$
\text { - } 15800-16400 \mathrm{~cm}^{-1} \text { (Fig. 5). }
$$

The sharp features shown in figure $5 \mathrm{~b}$ are reasonably well isolated from the broad bands which appear on each side in figure 5a. The spacing between lines $\alpha$ and $\beta$ in the axial spectrum is $13 \mathrm{~cm}^{-1}$ with a relative intensity $I_{\alpha} / I_{\beta} \sim 4-5$, depending upon the sample. The

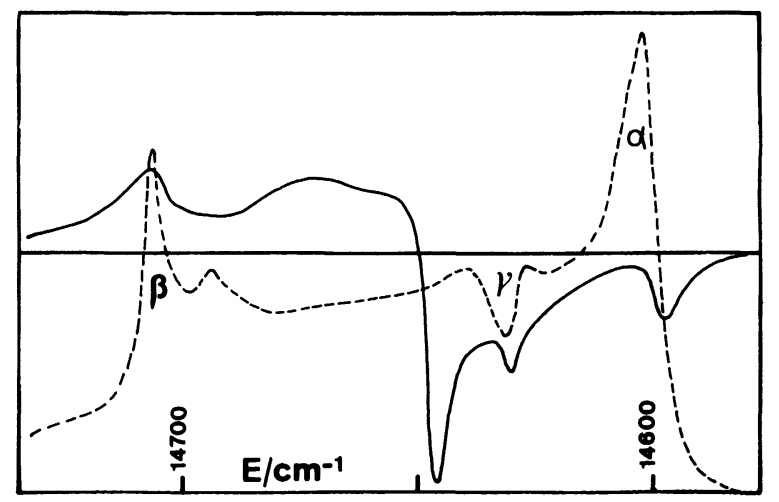

Fig. 3. - Axial absorption (-- -) and MCD (-) in the $14580-14730 \mathrm{~cm}^{-1}$ spectral range (arb. scales) at $1.7 \mathrm{~K}$. 


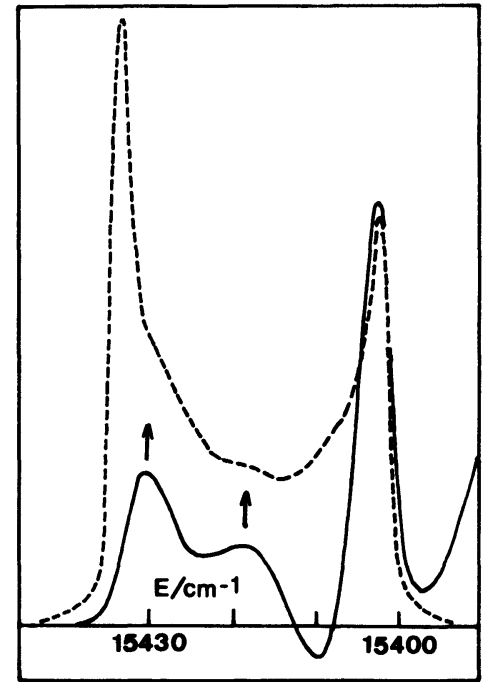

Fig. 4. - Axial absorption (--) and MCD (-) in the $15390-15450 \mathrm{~cm}^{-1}$ spectral range (arb. scales) at $2.1 \mathrm{~K}$.

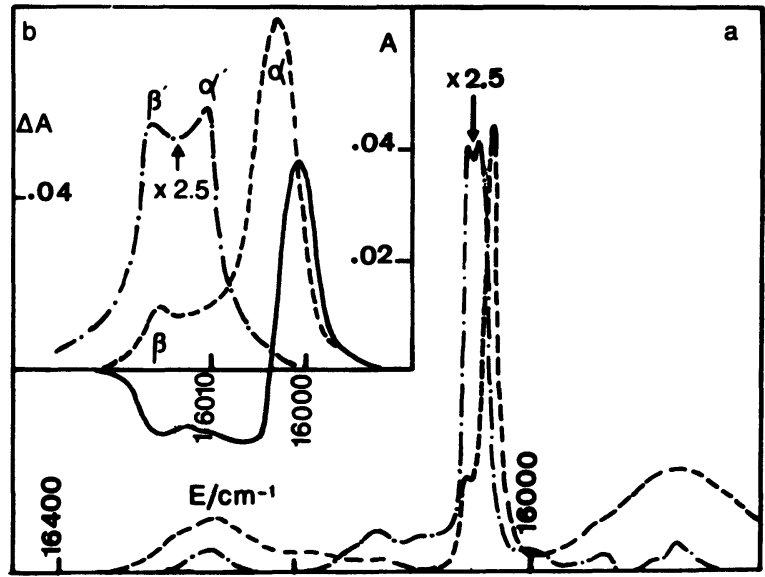

Fig. 5. - a) Axial (- -) and $\pi(-\cdot-)$ absorption spectra at $2.1 \mathrm{~K}$ in the $15800-16400 \mathrm{~cm}^{-1}$ spectral range; b) sharp lines around $16010 \mathrm{~cm}^{-1}$ with the accompanying MCD spectrum (-). Note that the $\pi$ spectrum is approximately twice as intense as the $\alpha$ spectrum.

shape of band $\alpha$ suggests that it covers two components. Under a $4.2 \mathrm{~T}$ longitudinal field, only this line shows a noticeable splitting.

The MCD profile for this region was found to vary only slightly from sample to sample, the peak corresponding to band $\beta$ eventually becoming more intense than that around $16007 \mathrm{~cm}^{-1}$ (Fig. 5b). We note that the negative part of the MCD becomes less negative $(\sim 10 \%)$ upon cooling the crystal from $4.2 \mathrm{~K}$ to $1.7 \mathrm{~K}$, in contrast to what we found during the same experiment for the $14580-14730 \mathrm{~cm}^{-1}$ region (slight increase in $\Delta A$ at all energies). These features suggest the existence of an impurity line in this region. This is further supported by the presence of a strong $\pi$ band (Fig. 5 b) which no theoretical calculation can reasonably account for and whose overall intensity, as compared to that of the axial spectrum, is greatly dependent upon the sample. It has in fact recently been established [14] that $U^{3+}$ is responsible for these features if $\mathrm{ThCl}_{4}$ is intentionally doped with this ion. Note that the stray $\pi$ band has a two-peak structure with $E_{\beta^{\prime}}-E_{\alpha^{\prime}}=7.5 \mathrm{~cm}^{-1}$ and intensities of ratio $I_{\beta^{\prime}} / I_{\alpha^{\prime}} \sim 0.97$.

$-17335-17390 \mathrm{~cm}^{-1}$ (Fig. 6).

The features in this region have been reproduced for several crystals with no noticeable spurious effects. No $\pi$ line is seen within several hundred wavenumbers on each side. The axial spectrum (Fig. 6a) shows three well resolved lines with $E_{\gamma}-E_{\alpha}=$ $32.5 \mathrm{~cm}^{-1}$ and $E_{\gamma}-E_{\beta}=20.5 \mathrm{~cm}^{-1}$. Our most important result is again that $A$ and $\Delta A$ vary by no more than about $3 \%$ between $4.2 \mathrm{~K}$ and $2.1 \mathrm{~K},\langle\Delta A\rangle_{0}$ being zero. Actually, as will be seen in $\S 4$, these very clear-cut data demonstrate that the ground state of $\mathrm{U}^{4+}$ in $\mathrm{ThBr}_{4}$ is $\Gamma_{4}\left(\mathrm{D}_{2 d}\right)$ and that the host has an incommensurate structure. Furthermore, since $\langle\Delta A\rangle_{0}$ $=0$, we know that we are dealing with a complete set of excited states, i.e., no noticeable mixing (via B) occurs with states outside this spectral range. The Zeeman spectrum under $4.2 \mathrm{~T}$ (Fig. $6 \mathrm{~b}$ ) is perfectly consistent with the MCD. Only line $\alpha$ shows a measurable splitting $\left(^{2}\right)(|g|=1.3)$ or displacement, the barycentre of the split lines (see arrow) being at an energy slightly higher than that of the zero field components. Similarly, the $\mathcal{A}_{1}$ term is also displaced towards high energies while only $\mathfrak{B}_{0}$ terms of opposite sign are seen for bands $\beta$ and $\gamma$, with a continuum between them. The difference in absorption profiles between figures $6 \mathrm{a}$ and $6 \mathrm{~b}$ results from the fact that

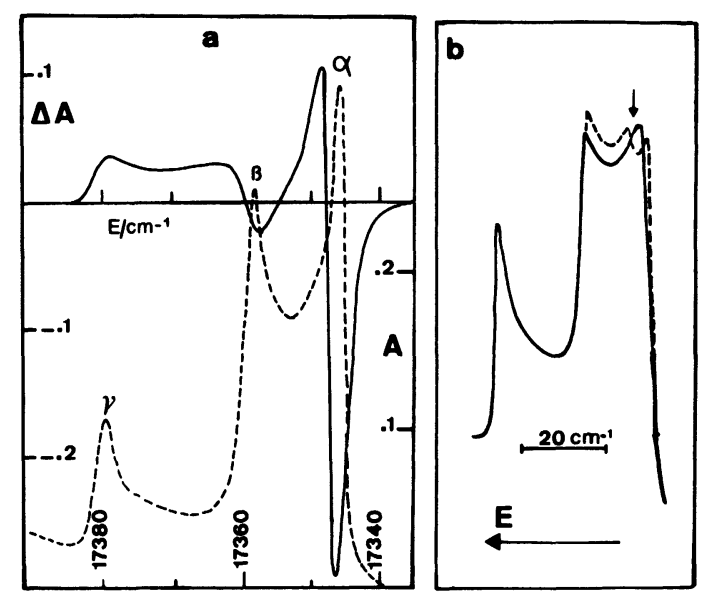

Fig. 6. - a) Axial (- - ) and MCD spectra through the $17335-17390 \mathrm{~cm}^{-1}$ spectral range at $2.1 \mathrm{~K}$; b) transmission spectra in zero field $(-)$ and under a $4.2 \mathrm{~T}$ longitudinal field (- - ).

$\left(^{2}\right)$ Note that our present definition of $g$ is different from that $\left(g^{\prime}\right)$ used in reference [7]. We have the relation $g^{\prime}=-2 g$. 
the Zeeman experiment was performed by simply recording the light transmitted by the sample.

$$
\text { - } 19300-19600 \mathrm{~cm}^{-1} \text { (Figs. 7-8). }
$$

The dominant feature of the axial spectrum (Fig. 7) is a three level reasonably sharp structure at low energy (see inset) with a less intense and broader vibrational counterpart roughly $50 \mathrm{~cm}^{-1}$ towards higher energies. The low temperature $(1.7 \mathrm{~K}) \pi$ spectrum shows also weak lines in this region, the sharpest occurring at $19311 \mathrm{~cm}^{-1}$.

The energies of the axial lines are such that $E_{\alpha}-E_{\gamma}=31.8 \mathrm{~cm}^{-1}$ and $E_{\alpha}-E_{\beta}=7.8 \mathrm{~cm}^{-1}$. The band $\beta$ is substantially broader than the two others and its intensity is greatly dependent upon the $\mathrm{U}^{4+}$ concentration of the sample. The MCD associated to these bands is weak and does not change appreciably upon cooling from $4.2 \mathrm{~K}$ to $1.7 \mathrm{~K}$.

An important result in that region is the occurrence of a disymmetrical hot band at 19311 (inset of Fig. 7).

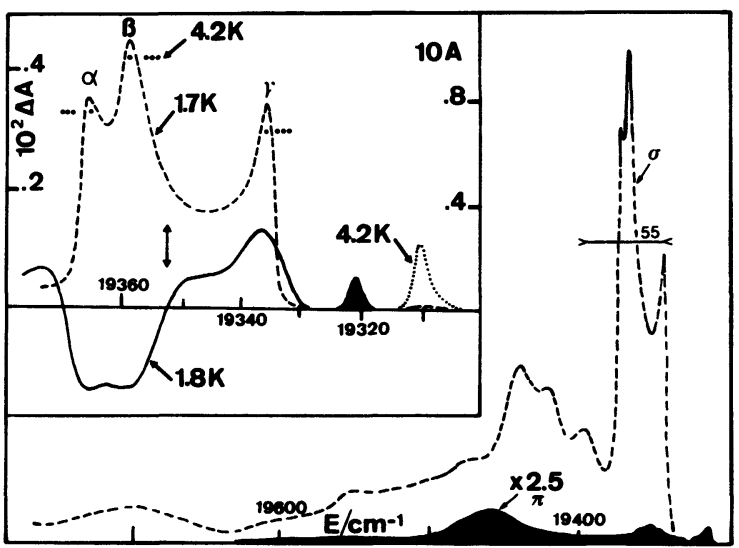

Fig. 7. - Axial (- . a at $2.1 \mathrm{~K}, \ldots$ at $4.1 \mathrm{~K})$ absorption, $\pi$ absorption (black $1.2 \mathrm{~K}$ ) and MCD at $2.1 \mathrm{~K}$ (full line in the inset) in the $19400 \mathrm{~cm}^{-1}$ region.

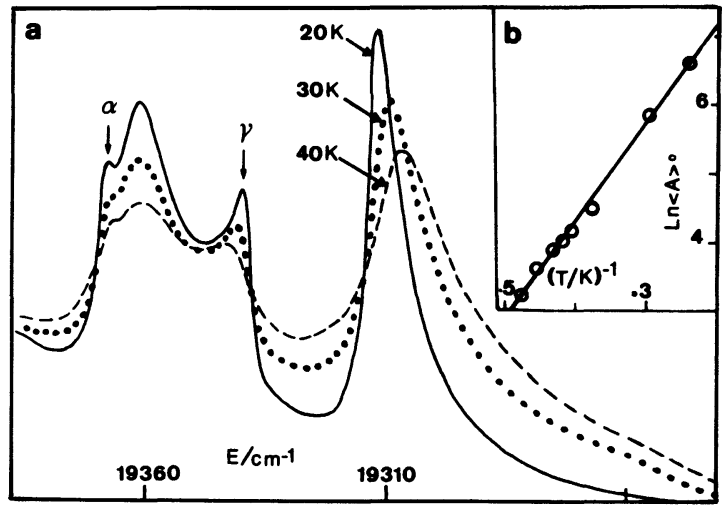

Fig. 8. - a) Cold band - hot band behaviour of absorption in the $19240-19400 \mathrm{~cm}^{-1}$ spectral range. $T / K=20(\square)$, $30(\ldots)$ and $40(--)$. b) Temperature dependence at $T \leqslant 4.2 \mathrm{~K}$ of the hot band peaking around $19310 \mathrm{~cm}^{-1}$.
It is observed at $4.2 \mathrm{~K}$ but shows no measurable MCD and nearly vanishes at $2.1 \mathrm{~K}$. The hot band-cold band behaviour is further illustrated in figure $8 \mathrm{a}$ at higher temperatures. It is clear that the hot band increases at the expanse of the cold band when $T$ increases, the former becoming less and less symmetrical. We made a detailed study of the $T$ dependence of $\langle A\rangle_{0}$ for the hot band at $T \leqslant 4.2 \mathrm{~K}$. The zero order moment was found to vary as $\exp (-W / k T)$ with $W=(9.8 \pm$ $0.2) \mathrm{cm}^{-1}$, as shown in figure $8 \mathrm{~b}$. In the frame of a $\mathrm{D}_{2 d}$ model, this finding demonstrates that a level lies $10 \mathrm{~cm}^{-1}$ above the ground state, both having the same degeneracy $d$. This spacing was found independently from fluorescence measurements [6]. It is also tempting to presume that the hot $\sigma$ band at $19311 \mathrm{~cm}^{-1}$ (intensity $I_{1}$ ) and the cold $\pi$ line at $19321 \mathrm{~cm}^{-1}\left(I_{0}\right)$ reach the same excited state. A glance at table I shows however that this possibility is excluded in $\mathrm{D}_{2 d}$ with either $d=1$ or $d=2$. We cannot explain either the hot band profile with a one-site picture. These failures provided a stimulus to find another type of explanation. We note finally that $I_{1} / I_{0}$ can be obtained easily from the zero order moments of the two bands. We found 1.45 at $4.2 \mathrm{~K}$, in excellent agreement with the value obtained at $20 \mathrm{~K}(1.40)$

$$
\text { - } 19 \text { 950-22 } 050 \mathrm{~cm}^{-1} \text { (Fig. 9). }
$$

Absorption features in these regions are significantly broader than those encountered so far. We assign two electronic $\sigma$ origins at 19966 and $21866 \mathrm{~cm}^{-1}$. Some plausible vibrational assignments

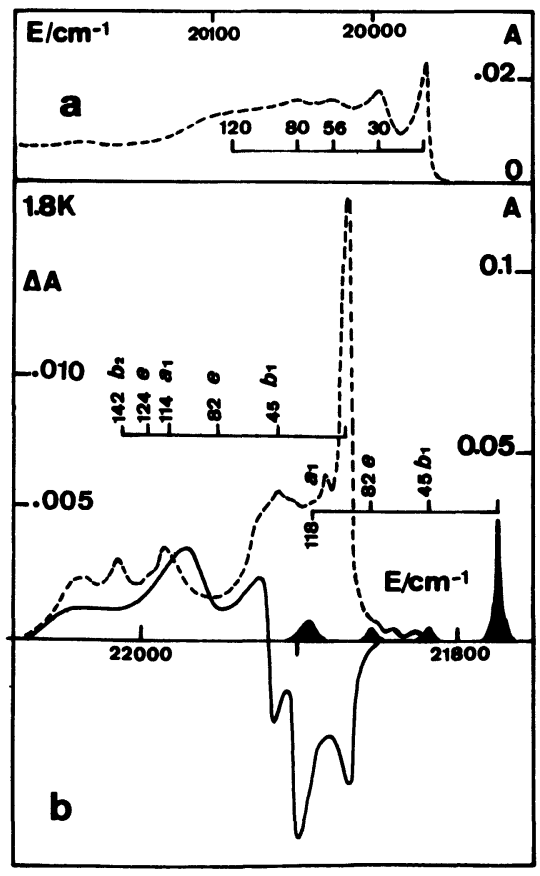

Fig. 9. - Axial absorption (- - $\pi$ absorption (black) and $\operatorname{MCD}(-)$ in the $19500-20150 \mathrm{~cm}^{-1}$ (a) and 21750 $22050 \mathrm{~cm}^{-1}$ (b) regions. All measurements at $1.8 \mathrm{~K}$. 
are sketched on the figure by reference to the Raman data [15]. No MCD could be measured in the 19 950-20 $150 \mathrm{~cm}^{-1}$ range. The MCD at higher energy (21 850-22 $\left.050 \mathrm{~cm}^{-1}\right)$ has an overall S-shape with $\langle\Delta A\rangle_{0} \sim 0$. A few weak $\pi$ lines were seen in that region for the more concentrated crystals.

\section{- Other $\pi$ features (Fig. 10).}

The sharpest band in the $\pi$ spectrum of $\mathrm{U}^{4+}$ occurs around $14369 \mathrm{~cm}^{-1}$ with two singularities separated by $3.25 \mathrm{~cm}^{-1}$. The low energy peak is about $3 \%$ more intense that the high-energy one. This band is accompanied by a $\sigma$ hot band at $14359 \mathrm{~cm}^{-1}$ having the same profile and temperature behaviour as that found at $19311 \mathrm{~cm}^{-1}$. Note that the $10 \mathrm{~cm}^{-1}$ spacing is met for the second time in these experiments. A similar situation occurs at a somewhat higher energy. A hot $\pi$ band is seen at $14609 \mathrm{~cm}^{-1}$ on the foot of a residual $(\sim 2 \%) \sigma$ cold spectrum peaking around $14620 \mathrm{~cm}^{-1}$.

Finally, a much weaker $\pi$ line with two singularities was observed around $15212 \mathrm{~cm}^{-1}$ (Fig. 10). It occurs on the blue foot of an intense and highly structured broad band. On the basis of this experiment only, it is clearly impossible to locate precisely an origin for the latter.

\section{- Summary.}

Anticipating the fact $(\S 4)$ that all sharp absorption structures with two $(\pi)$ or two/three $(\sigma)$ singularities actually cover only one electronic origin in $\mathrm{D}_{2 d}$, we have summarized our results for energy, polarization and dipole strengths in the first three columns of table II. The figures quoted are sometimes slightly different from those published previously [7], due to the use of several spectrometers.

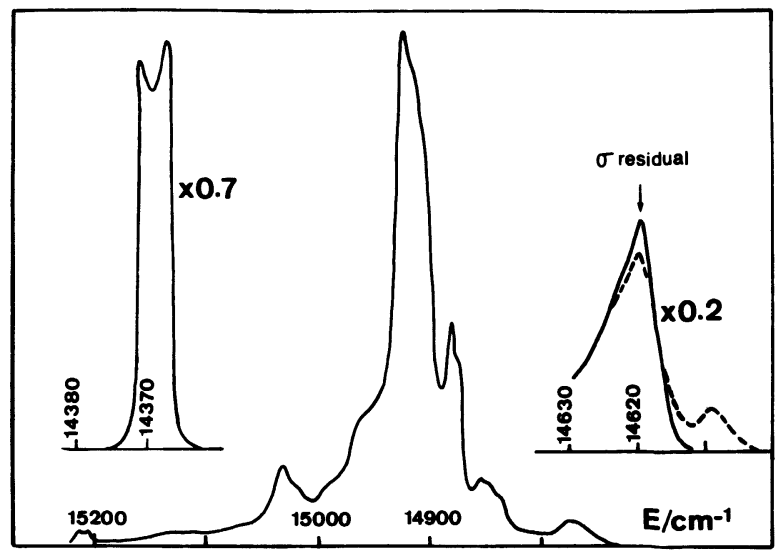

Fig. 10. - Most important features in the $\pi$ spectra at $2.1 \mathrm{~K}$ $(\longrightarrow)$ and $4.2 \mathrm{~K}(--)$.

In view of the arguments developed so far, it should be realized that an accurate numbering of these origins has been long and difficult. Some of the assignments (marked by an asterisk) could only be firmly established via fluorescence and site selective excitation experiments [6].

3.3 ROLE OF IMPURITIES. - The presence of impurities in our crystals was most clearly demonstrated by MCD experiments. For example, all the features marked by arrows on figure 11 are spurious on the basis of their sample dependence. Actually, only the two peak structure around $15400 \mathrm{~cm}^{-1}$ was retained as belonging to $\mathrm{U}^{4+}$ in the $15100-15500 \mathrm{~cm}^{-1}$ region. The rest of the MCD spectrum shows some low temperature dependence which requires further investigation. For example, the term around

Table II. - Experimental (e) and theoretical ( $\mathrm{t}$, from Ref. [7]) results in the investigated spectral range. Eigenvectors are given with the relative importance of the dominant $\mid S L J>$ levels.

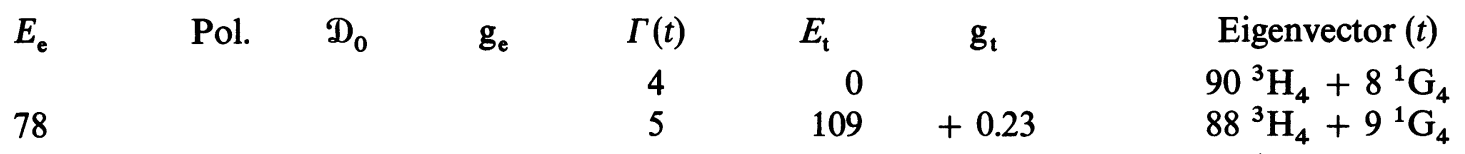

\begin{tabular}{|c|c|c|c|c|c|c|c|}
\hline 14370 & $\pi$ & & & 1 & 14392 & & $41{ }^{3} \mathrm{P}_{0}+19{ }^{1} \mathrm{D}_{2}+17{ }^{3} \mathrm{P}_{2}$ \\
\hline 14650 & $\sigma$ & 600 & +0.58 & 5 & 14632 & +1.05 & $48{ }^{1} \mathrm{D}_{2}+35{ }^{3} \mathrm{P}_{2}$ \\
\hline $14890(*)$ & $\pi$ & & & 1 & 14940 & & $45{ }^{3} \mathbf{P}_{0}+27{ }^{1} \mathrm{D}_{2}+19{ }^{3} \mathrm{P}_{2}$ \\
\hline 15212 & $\pi$ & & & 1 & 15229 & & $47^{3} \mathrm{~F}_{4}+40{ }^{1} \mathrm{G}_{4}$ \\
\hline 15420 & $\sigma$ & 29 & ? & 5 & 15389 & +0.97 & $47^{1} \mathrm{G}_{4}+48^{3} \mathrm{~F}_{4}$ \\
\hline $15828\left(^{*}\right)$ & $\pi$ & & & 1 & 15821 & & $48{ }^{1} G_{4}+41{ }^{3} F_{4}$ \\
\hline 16003 & $\sigma$ & 16 & -1.18 & 5 & 16052 & -1.13 & $51{ }^{1} \mathrm{G}_{4}+42{ }^{3} \mathrm{~F}_{4}$ \\
\hline 17347 & $\sigma$ & 100 & +1.28 & 5 & 17313 & +1.50 & $97{ }^{3} \mathrm{P}_{1}$ \\
\hline 19321 & $\pi$ & & & 1 & 19285 & & $88{ }^{1} \mathrm{I}_{6}+8{ }^{3} \mathrm{H}_{6}$ \\
\hline 19352 & $\sigma$ & 52 & -0.05 & 5 & 19344 & 0 & $91{ }^{1} \mathrm{I}_{6}+8{ }^{3} \mathrm{H}_{6}$ \\
\hline 19966 & $\sigma$ & & $?$ & 5 & 19991 & +1.00 & $93{ }^{1} \mathbf{I}_{6}+7{ }^{3} \mathrm{H}_{4}$ \\
\hline $20360\left(^{*}\right)$ & $\sigma$ & & ? & 5 & 20387 & +2.05 & $92{ }^{1} \mathrm{I}_{6}+6{ }^{3} \mathrm{H}_{6}$ \\
\hline 20408 & $\pi$ & & & 1 & 20446 & & $91{ }^{1} \mathrm{I}_{6}+5{ }^{3} \mathrm{H}_{6}$ \\
\hline 21866 & $\sigma$ & & +1.59 & 5 & 21813 & +1.32 & $61{ }^{3} P_{2}+35{ }^{1} D_{2}$ \\
\hline 22220 & $\pi$ & & & 1 & 22229 & & $57{ }^{3} P_{2}+37{ }^{1} D_{2}$ \\
\hline
\end{tabular}




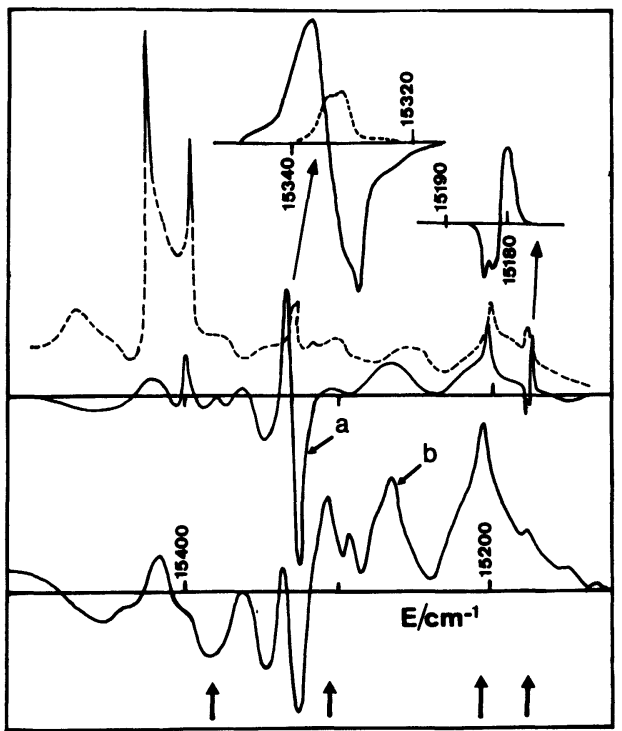

Fig. 11. - Role of impurities in the $15100-15500 \mathrm{~cm}^{-1}$ spectral range. MCD experiments (full lines) were run on two different samples with a different band pass for (a) and (b) $(0.3 \AA$ and $0.6 \AA$ respectively). The absorbance is shown in dotted lines.

$15335 \mathrm{~cm}^{-1}$ becomes less symmetrical upon cooling from $4.2 \mathrm{~K}$ to $1.8 \mathrm{~K}$, this implying that the responsible impurity is paramagnetic.

A large number of weak sharp lines were observed in absorption for the more concentrated crystals on the red foot of the strongest $\sigma$ band, i.e., between 14350 and $14550 \mathrm{~cm}^{-1}$. These are shown with moderate resolution in figure 12a with the associated MCD (Fig. 12b). Under the appropriate resolution $\left(0.6 \mathrm{~cm}^{-1}\right)$, the MCD shows a wealth of fine structure (Fig. 12c). This was examined in detail as a function of $T$ for the strongest lines in the $14430-14450 \mathrm{~cm}^{-1}$ range (Fig. 12d). We found four absorption components, each being associated with a combination of an $\mathcal{A}_{1}$ term and a $\mathrm{C}_{0}$ term. We conclude therefore that these lines are associated with a paramagnetic impurity.

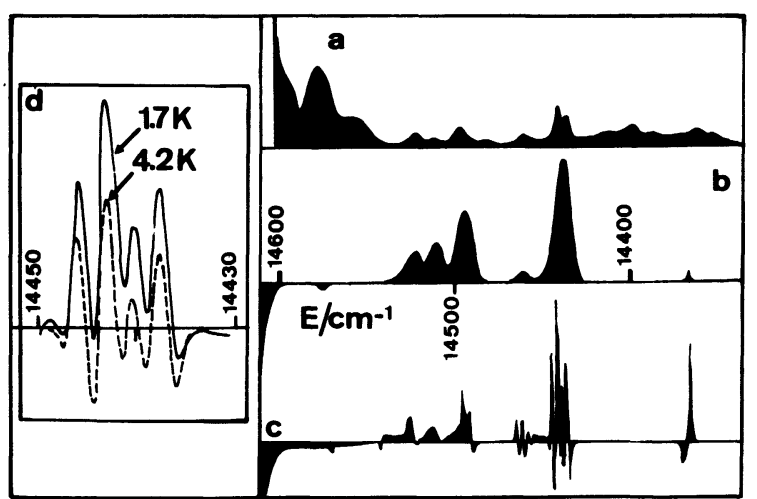

Fig. 12. - Role of impurities in the $14350-14600 \mathrm{~cm}^{-1}$. range. $\mathrm{a}$ and $\mathrm{b}: A$ and $\Delta A$ at moderate resolution; $\mathrm{c}$ and $\mathrm{d}$ : $\Delta A$ at high resolution.
In view of their sharpness, it has to be an actinide or lanthanide ion, most likely $\mathrm{U}^{3+}$. Four $\pi$ lines in the region $13710-13725 \mathrm{~cm}^{-1}$ probably belong to the same species.

\section{Discussion.}

\subsection{SPECTROSCOPIC ASSIGNMENTS.}

4.1.1 Historical. - The structure of the ground multiplet of $\mathrm{U}^{4+}$ in $\mathrm{D}_{2 d}$ symmetry (zircon and $\mathrm{ThBr}_{4}$ hosts) has long been uncertain due to the lack of conclusive experiments. Calculations can produce either a singlet or a doublet ground state for the $\mathrm{f}^{2}$ configuration. Actually, it was precisely to firmly establish this characteristic that the present work was undertaken.

In 1975 [3], Zeeman and MCD data on $\mathrm{ZrSiO}_{4} / \mathrm{U}^{4+}$ proved unambiguously that the ground state is $\Gamma_{4}$ for that host. At present, the accepted picture for $\mathrm{ThBr}_{4} / \mathrm{U}^{4+}$ is also $\Gamma_{4}$ lowest $\mathrm{D}_{2 d}$. Our arguments for this choice can be summarized as follows :

(i) our Zeeman effect experiments have shown that the splitting is substantially different on the various lines which have been examined carefully. This necessarily implies a singlet ground state since doublet $\leftrightarrow$ doublet transitions are forbidden for non-Kramers ions in $\mathrm{D}_{2 d}$. Due to the use of linearly polarized light however, we lack one crucial piece of information, i.e., the respective circular polarization of the split components ;

(ii) the choice of $\Gamma_{4}$ rests essentially upon a precise numbering of the $\sigma$ and $\pi$ electronic origins. In the $14000-22200 \mathrm{~cm}^{-1}$ region investigated here, we should find those crystal field levels originating from the strongly mixed free ion terms ${ }^{3} \mathrm{P}_{0},{ }^{1} \mathrm{D}_{2},{ }^{1} \mathrm{G}_{4},{ }^{3} \mathrm{P}_{1}$ ${ }^{1} \mathrm{I}_{6}$ and ${ }^{3} \mathrm{P}_{2}$. Altogether, one expects $7,2,5,5$ and 7 levels of $\Gamma_{1} \ldots \Gamma_{5}$ symmetry respectively. With the help of table Ia, one can easily predict the number of $\sigma$ and $\pi$ lines expected, according to the nature of the ground state in $\mathrm{D}_{2 d}$ (cf. Table III). Experimentally (Table II) we found $8 \sigma$ lines and $7 \pi$ lines and this strongly suggests that $\Gamma_{4}$ is indeed the ground state. This assignment is further supported by the observance of a $\pi$ band with two singularities associated with ${ }^{3} \mathrm{P}_{0}\left(\Gamma_{1}\right)$ around $14400 \mathrm{~cm}^{-1}$. In no case can the lowest state be $\Gamma_{5}$ (too many $\sigma$ lines predicted) or $\Gamma_{3}$ (too few).

Table III. - Number of predicted $\sigma$ and $\pi$ lines according to the nature of the ground state.

$\begin{array}{crc}\text { Ground state } & N_{\sigma} & N_{\pi} \\ - & - & 5 \\ \Gamma_{1} & 8 & 5 \\ \Gamma_{2} & 8 & 2 \\ \Gamma_{3} & 8 & 7 \\ \Gamma_{4} & 8 & 8 \\ \Gamma_{5} & 19 & \end{array}$




\subsubsection{New information.}

(i) Nature of the ground state. - A definite proof of the ground state being $\Gamma_{4}$ for $\mathrm{U}^{4+}$ in $\mathrm{ThBr}_{4}$ is provided by the present MCD data, as will be demonstrated now. Within the assumption of a $\mathrm{D}_{2 d}$ model, $\Gamma_{5}$ is excluded because none of the observed features in the spectra show the $\mathrm{C}_{0} / k T$ behaviour observed previously for all paramagnetic systems. For example, the $\Delta A$ associated to band $\gamma$ in figure 6 has indeed the shape of the corresponding absorption component but $\Delta \boldsymbol{A} / \boldsymbol{A}$ does not vary within the limits of experimental errors $(\sim 1 \%)$ between $4.2 \mathrm{~K}$ and $2.1 \mathrm{~K}$.

All calculations made so far predict a fairly well isolated $D_{2 d}$ doublet with almost pure ${ }^{3} P_{1}$ parentage around $17300 \mathrm{~cm}^{-1}$. With our convention, $g$ is certainly positive for that state since the $M_{J}=+1$. Zeeman component transforms as $\Gamma_{3}^{\prime}$ in $S_{4}$ symmetry. Then, for a centre in $\mathrm{D}_{2 d}$ symmetry, the $\mathcal{A}_{1}$ parameter associated with the transition should be positive or negative according to whether the ground state is $\Gamma_{2}^{\prime}$ or $\Gamma_{1}^{\prime}$ in $S_{4}(\S 2)$. Actually, the argument applies as well to the first order moment $\langle\Delta A\rangle_{1}$ if the integration is carried over the features associated to all $D_{2}$ and $D_{2 d}$ centres in the corresponding region. The only assumption is that the distortion is sufficiently small to act as a perturbation, i.e., it does not $\mathrm{mix}{ }^{3} \mathbf{P}_{1}$ with any other level. This turns out to be the case since $\langle\Delta A\rangle_{0}$ is found to be zero. From our results in figure 6, it is clear that both the $\mathcal{A}_{1}$ term at low energy and $\langle\Delta A\rangle_{1}$ over the entire band are positive. We therefore conclude that the ground state is $\Gamma_{2}^{\prime}$ in $\mathrm{S}_{4}$, i.e., $\Gamma_{3}$ or $\Gamma_{4}$ in $\mathrm{D}_{2 d}$. It has to be $\Gamma_{4}$ since $\Gamma_{3}$ can be firmly ruled out on the basis of the lack of any noticeable $\pi$ absorption $\left(\Gamma_{3} \rightarrow \Gamma_{2}\right.$ of $\left.{ }^{3} P_{1}\right)$ in this region and the large number of observed $\pi$ components in the whole spectrum, as compared to only 2 predicted (Table III) in this situation. The lack of $\pi$ absorption also rules out the possibility of $\mathrm{C}_{2}$ symmetry in the low temperature phase.

(ii) Testing of wavefunctions. - With a $\Gamma_{4}$ ground state for $\mathrm{D}_{2 d}, \sigma$ and $\pi$ transitions are allowed towards excited states of symmetry $\Gamma_{5}$ and $\Gamma_{1}$ respectively. One therefore arrives at the assignments shown in table II which contains also the results of most recent crystal field calculation [7] (energies and $g$ factors). As can be seen, the computed energies match those observed fairly well.

MCD also provides a powerful means to test the wavefunctions. $g$ factors were determined experimentally from first order moments. As shown in table II, not only does the calculation account for the actual sign of $g$ for 5 bands, but its magnitude is also reasonably well reproduced. The uncertainty on experimental data is estimated to be about $10 \%$.

$g$ unfortunately could not be measured for the bands at $19966 \mathrm{~cm}^{-1}$ and $20360 \mathrm{~cm}^{-1}$ (Fig. 9a) because the absorbance in these regions was too low on the investigated samples. It should be remembered that $\Delta A$ is grossly proportional to the maximum absorbance, this being typically $10^{-2}$ at $19966 \mathrm{~cm}^{-1}$ and $10^{-3}$ at $20360 \mathrm{~cm}^{-1}$ for a crystal of thickness $0.54 \mathrm{~mm}$ and of concentration $\mathrm{U} / \mathrm{Th}=2 \times 10^{-4}$. The $\mathcal{A}_{1}$ coefficient measured for the band at $15420 \mathrm{~cm}^{-1}$ (Fig. 4) is not reported in table II. It is unreliable since we have demonstrated that sample dependent (impurity) features occur in that region.

4.2 INCOMMENSURATE MODEL. - The model presented in detail at the beginning of this paper provides a nice understanding of most of the unusual features observed in absorption and MCD experiments. Hereafter, we shall use some now well established facts in our fitting procedure. Of course the ground state is singlet $\Gamma_{4}$ in $D_{2 d}$. Furthermore, fluorescence experiments [7] can only be understood if the next higher level is $\Gamma_{5}\left(D_{2 d}\right)$ at $78 \mathrm{~cm}^{-1}$, the splitting of its two $D_{2}$ branches implying $v_{1}^{1}=69 \mathrm{~cm}^{-1}$ and $v_{2}^{1}=2 \mathrm{~cm}^{-1}$. In the following, we shall use $v_{1}$ to characterize the excited state of the transition considered.

4.2.1 Absorption and MCD profiles. - First, we select the ${ }^{3} \mathrm{P}_{1}$ region around $17360 \mathrm{~cm}^{-1}$ since it is fairly well isolated and no spurious effects were observed. Our simulated curves (for $v_{2} / v_{1}=4.3$ ) are shown on figure 13. A comparison with figure 6 indicates that the overall experimental profiles are well reproduced. Clearly, as shown by the Zeeman and MCD curves, it is not the $\alpha$ band which splits under $B$ but the component associated with the site at $\varphi=0$ and located slightly higher in energy than $\alpha$. The relative intensities of the three absorption singularities are well reproduced if we take a due account of the intense absorption background illustrated in figure 2.

During the preliminary work of Delamoye [5] the reasonable assumption was made that $v_{1}(\sim \eta)$ was probably larger than $v_{2} / 2\left(\sim \eta^{2}\right)$ and this led to the conclusion that only two singularities should be observed in both the $\sigma$ and the $\pi$ spectra. It is thus striking to see that a good agreement between prediction and observation occurs precisely for a band for which $\left(2 v_{1} / v_{2}\right)>2$. Actually, we know nothing of the coefficients of $\eta$ and $\eta^{2}$ and there may occur the

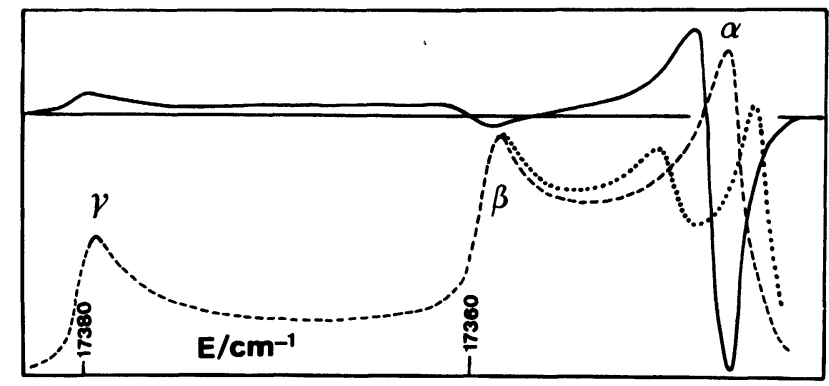

Fig. 13. - Simulation of the MCD (-), axial absorption in zero field (- - ) and axial absorption under a $4.2 \mathrm{~T}$ field (...). The value of $g$ for the excited state is $1.28 . T=1.8 \mathrm{~K}$, $v_{1}=10.25, v_{2}=44$ and $\gamma=0.8 \mathrm{~cm}^{-1}$. 
unusual situation where the latter is much bigger than the former. This is not really surprising since, in the case of a singlet $\rightarrow$ doublet transition [5], the odd $\left(v_{1}, v_{3} \ldots\right)$ and even $\left(v_{2} \ldots\right)$ terms do not arise via the same process, i.e., we do not merely have a Taylor expansion.

The features in the $16000 \mathrm{~cm}^{-1}$ region (Fig. 5) were simulated with $v_{1}=6.5 \mathrm{~cm}^{-1}$ and $v_{2} / v_{1} \sim 3\left(\gamma=1 \mathrm{~cm}^{-1}\right)$. The agreement was found to be satisfactory for the MCD while the calculated value $I_{\alpha} / I_{\beta}$ is smaller $(\sim 3)$ than that found experimentally (4-5). This failure is quite certainly related to the presence of a spurious very strong $\pi$ line and a positive $\mathcal{C}_{0}$ residual contribution in the $16007-16015 \mathrm{~cm}^{-1}$ region (Fig. 5). Both are tentatively assigned to a $\Gamma_{7} \rightarrow \Gamma_{6}$ transition (allowed $\pi$ and $\sigma$ ) of $\mathrm{U}^{3+}$ (possibly of charge transfer character).

Apart from the weak bumps shown by arrows in figure 4, the absorption spectrum in the $15400 \mathrm{~cm}^{-1}$ region is fairly well reproduced with $v_{1}=15 \mathrm{~cm}^{-1}$, $v_{2}=-6 \mathrm{~cm}^{-1}$ and $\gamma=0.6 \mathrm{~cm}^{-1}$. The MCD data are not and this is due to the mentioned presence of presumably paramagenetic impurities giving a relatively large MCD. The sharp $\pi$ line at $14370 \mathrm{~cm}^{-1}$ (Fig. 10) is well reproduced with $v_{2}=-7.5 \mathrm{~cm}^{-1}$. Note that this figure actually corresponds to the difference in $v_{2}$ coefficients for the excited state and the ground state. The negative sign is imposed by the fact that the low energy component is approximately $3 \%$ more intense than the high energy one:

The profile and $T$ dependence of hot bands (e.g., Fig. 8) is also produced by our calculation, as shown in figure 14. Curve a $\left(\gamma=0.8 \mathrm{~cm}^{-1}\right)$ is similar to our experimental spectrum (Fig. 7) while b shows the red shift and red broadening of the line under heating.

We now consider the $19300 \mathrm{~cm}^{-1}$ region (Fig. 7). No value of $v_{2} / v_{1}$ could possibly reproduce the absorption ( 3 bands) and MCD spectra. Regarding

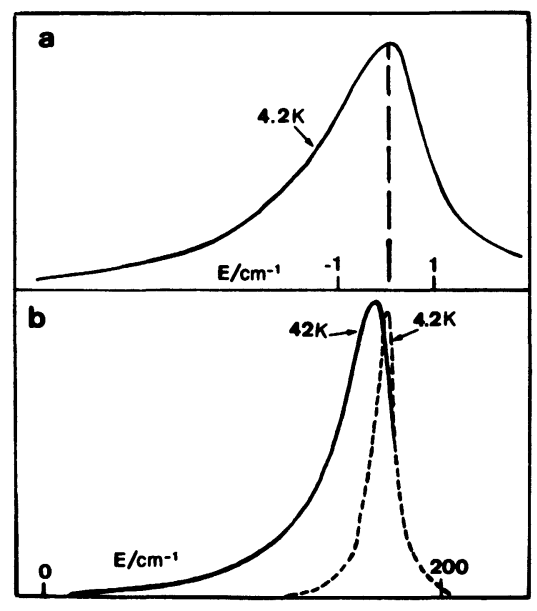

Fig. 14. - Simulation of the hot bands : (a) proper profile at $4.2 \mathrm{~K}$ of the band at $19311 \mathrm{~cm}^{-1}\left(\gamma=0.8 \mathrm{~cm}^{-1}\right)$; (b) temperature dependence of the profile (we used $\gamma=7 \mathrm{~cm}^{-1}$ to save computing time). the former, it is clear from figure 1 that band $\alpha$ would be much stronger than band $\gamma$ if $\beta$ was a " normal " third band. Regarding the latter, we do observe the expected opposite $\mathscr{B}_{0}$ terms for $\alpha$ and $\gamma$ but the plausible $\mathcal{A}_{1}$ term around $19352 \mathrm{~cm}^{-1}$ (arrow) is surprisingly small. Such a result is only obtainable when the half band width is larger than $v_{1}$, a quite unrealistic situation. Actually, calculations (Table II) indicate that the $g$ factor should be close to zero and it is therefore likely that the whole MCD can hardly be attributed to only $\mathrm{U}^{4+}$. Note that " extra » bands are also found in a few regions of the infra-red spectrum of $\mathrm{ThBr}_{4} / \mathrm{U}^{4+}$.

Difficulties also arise in the $14650 \mathrm{~cm}^{-1}$ region (Fig. 3). The model accounts for the respective intensities of bands $\alpha$ and $\beta$ by placing $E_{0}$ at $14650 \mathrm{~cm}^{-1}$ where a disymmetrical $\mathcal{A}_{1}$ term seems to occur, and choosing $v_{1}=46 \mathrm{~cm}^{-1}, v_{2}=5 \mathrm{~cm}^{-1}$. This interpretation is further supported by the fact (Fig. 15) that the $\alpha-\beta$ splitting ( $\sim$ order parameter $\eta$ ) is experimentally found to vary as $\left(T-T_{\mathrm{c}}\right)^{1 / 3}$ for $T \leqslant 40 \mathrm{~K}$, as does the frequency of the Raman soft mode [15]. Note that an exactly similar result was obtained for the $\alpha-\beta$ spacing in figure 8 . This $1 / 3$ figure is quite reliable since it was obtained by taking a proper account of the variation of the bandwidth with temperature. No reliable measurement of the splitting between bands $\alpha$ and $\beta$ at temperatures near $T_{\mathrm{c}}=95 \mathrm{~K}$ could be made because of the vibrational broadening of the lines upon heating. The $\left(T_{c}-T\right)^{1 / 3}$ behaviour of $\eta$ far below $T_{\mathrm{c}}$ is not presently understood.

Returning to figure 3 , our model certainly fails to account for the additional bumps and the dip $\gamma$ in the absorption spectrum, as well as for some of the MCD structures.

4.2.2 Role of vibrations. - One possible issue to the puzzling questions raised for the above two regions is to consider the role of vibrations, which we have ignored so far in our discussion. As encountered for $d^{n}$ ions at a field of $\mathrm{D}_{2 d}$ symmetry [16], electronic states are generally coupled to several vibrational modes. This obviously occurs in figure 7 where the absorption features around $19400-19450 \mathrm{~cm}^{-1}$ bear a striking resemblance to the three-bands origin.

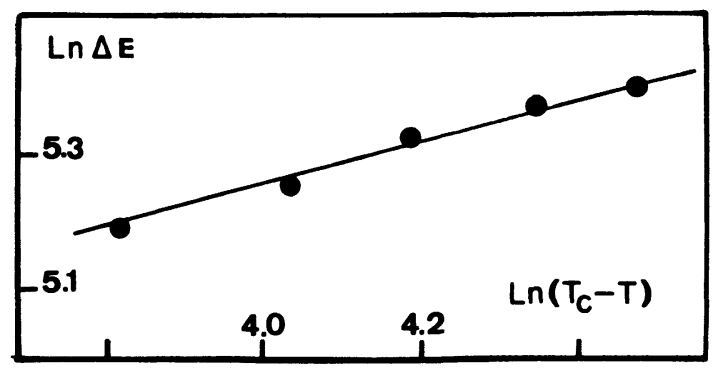

Fig. 15. - Plot of the splitting between bands $\alpha$ and $\beta$ in figure 3 as a function of $T_{c}-T$ on a logarithmic scale. 
The active modes might be $b_{1}$ at $45 \mathrm{~cm}^{-1}$ and $b_{2}$ at $66 \mathrm{~cm}^{-1}$ [15]. The consequences of vibrational coupling are also fairly obvious in figure 9. Finally, Raman experiments at $4.2 \mathrm{~K}$ reveal modes at an even lower frequency : $30-38 \mathrm{~cm}^{-1}$ and $19 \mathrm{~cm}^{-1}$ (soft mode). We think it therefore likely that vibrations play a very significant role when their frequency is smaller than (or comparable to) $2 v_{1}$, as, e.g., around $14650 \mathrm{~cm}^{-1}$. Although the exact result is difficult to predict, it is fairly certain that anticrossing of $\mathrm{D}_{2}$ states may occur in this situation if for example the $\left|\Gamma_{2}\right\rangle$ and $\left|\Gamma_{4}\right\rangle$ electronic components of a $\mathrm{D}_{2 d}$ doublet couple to an $\mathrm{a}_{2}$ or $\mathrm{b}_{2}\left(\mathrm{D}_{2 d}\right)$ mode $\left(\Gamma_{3}\right.$ in $\left.\mathrm{D}_{2}\right)$ to produce $\left.\left|\Gamma_{4}\right\rangle\right\rangle$ and $\left|\Gamma_{2}\right\rangle$ vibronic states. Thus $\left|\Gamma_{2}\right\rangle$ and $\left|\Gamma_{2}\right\rangle$ or $\left|\Gamma_{4}\right\rangle$ and $\left|\Gamma_{4}\right\rangle$ will interact strongly and this will lead to a dip in the absorption spectrum. The separation between the two arrows in figure $3\left(\sim 10 \mathrm{~cm}^{-1}\right)$ is just twice the magnitude of the matrix element between the two mixing states. Additional bands will occur for $\varphi= \pm \pi / 2$, due to the presence of vibrational branches. Our arguments are equally valid in the case of a coupling with an $a_{1}$ mode (e.g., the soft mode). Anticrossing then occurs between the two lowest of the four $\mathrm{D}_{2}$ branches.

4.2.3 Is there a phase pinning ? - At least for our crystal of concentration $c \sim 2 \times 10^{-4}$ (U/Th), our data demonstrate that there is no pinning of the incommensurate modulation at $\varphi=0$. This would be observable via MCD in several regions of the spectrum. We made some calculations by increasing the number of sites at $\varphi=0$. Invariably, the $\mathcal{A}_{1}$ term in the MCD becomes quickly symmetrical, all other features being completely swamped out. This prediction contrasts strongly with our observations, non only around $17345 \mathrm{~cm}^{-1}\left({ }^{3} \mathrm{P}_{1}\right.$ region) but also around $16003 \mathrm{~cm}^{-1}$ (Fig. 6). The argument is independent of the type of distribution adopted around $\varphi=0$, e.g., $\delta$-function or Gaussian. If the pinning occurred for $\varphi \neq 0$, then it would show up as extra peaks in all spectral regions, i.e., ${ }^{3} \mathbf{P}_{1}$ among others.

\section{Conclusions.}

A number of firm conclusions arise from this paper. First of all, MCD experiments leave no doubt that the ground state of $\mathrm{U}^{4+}$ in $\mathrm{ThBr}_{4}$ is indeed $\Gamma_{4}$ in $\mathrm{D}_{2 d}$ symmetry. New information has been gained regarding the $g$ factors (magnitude and sign) of five excited states. Our findings strongly support the results of recent crystal field calculations. MCD has also demonstrated quite neatly that the investigated samples contained one (or several) paramagnetic impurity, this being $\mathrm{U}^{3+}$ in all likehood.

The model presented previously for the low-temperature phase $\left(D_{2 d}-D_{2}\right.$ symmetry) has been developed quantitatively further and extended to the simulation of Zeeman and MCD spectra. It is found that our simulations and experiments are in good agreement in the ${ }^{3} \mathrm{P}_{1}$ region. From this result, we are able to state that : (i) the phase is probably truly incommensurate since an inferior limit to the number of centres is found to be 80 ; (ii) for a low concentration of $\mathrm{U}^{4+}\left(2 \times 10^{-4}\right)$, our data demonstrate the absence of a phase pinning at either $\varphi=0$ or $\varphi \neq 0$; (iii) for $T<40 \mathrm{~K}$, the order parameter $\eta$ is found to vary as $\left(T_{\mathrm{c}}-T\right)^{1 / 3}$, as does the frequency of the Raman soft mode. This behaviour far below $T_{\mathrm{c}}=95 \mathrm{~K}$ is not presently understood.

Two regions of the spectra (19 360 and $14600 \mathrm{~cm}^{-1}$ ) are not yet fully understood although we believe that electron phonon coupling in excited states plays an important role. Further progress could arise from a detailed investigation of $\mathrm{ThCl}_{4} / \mathrm{U}^{4+}$ (change of $T_{c}$ and the vibrational frequencies) or $\mathrm{ThBr}_{4}\left(\mathrm{ThCl}_{4}\right)$ intentionally doped with the impurity $\mathrm{U}^{3+}$. We note finally that our model is easily applicable to Kramers ions in hosts with an incommensurate phase.

References

[1] Genet, M., Delamoye, P., Edelstein, N. and Conway, J., J. Chem. Phys. 67 (1977) 1620.

[2] Richman, I., Kislink, P. and Wong, E. Y., Phys. Rev. 155 (1967) 262.

[3] Mackey, D. J., Runciman, W. A. and Vance, E. R., Phys. Rev. B 11 (1975) 211.

[4] Bernard, L., Currat, R., Delamoye, P., Zeyen, C. M. E., Hubert, S. and De KouchKovSKy, R., J. Phys. C : Solid State Phys. 16 (1983) 433.

[5] Delamoye, P. and Currat, R., J. Physique Lett. 43 (1982) L-655.

[6] Delamoye, P., Krupa, J. C., Conway, J. and Edelstein, N., Phys. Rev. B 28 (1983) 4913.

[7] Delamoye, P., Rajnak, K., Genet, M. and Edelstein, N., Phys. Rev. B 28 (1983) 4923.

[8] See, e.g., BRIAT, B., in Electronic States of Inorganic Compounds : New experimental techniques, edited by P. Day (Reidel) 1975.
[9] Koster, G. F., Dimmock, J. O., Wheeler, R. G. and STATZ, H., Properties of the thirty-two point groups (M.I.T. Press, Cambridge) 1963.

[10] Ferre, J., Boccara, A. C. and Briat, B., J. Physique 31 (1970) 631.

[11] Blinc, R., Aleksandrova, I. P., Chaves, A. S., Milia, F., Rutar, V., Seliger, J., Topic, B. and Zumer, S., J. Phys. C : Solid State Phys. 15 (1982) 547.

[12] Hussonois, M., Krupa, J. C., Genet, M., Brillard, L. and CARlier, R., J. Crystal Growth 51 (1981) 11.

[13] Canit, J. C. and Badoz, J., Appl. Opt. 23 (1984) 2861.

[14] KRUPA, J. C., personal communication.

[15] Hubert, S., Delamoye, P., Lefrant, S., Lepostollec, M. and Hussonnors, M., J. Solid State Chem. 36 (1981) 36.

[16] Briat, B. and Canit, J. C., Mol. Phys. 48 (1983) 33. 